\title{
Basic principles in the management of thermal injuries
}

\author{
Karpelowsky JS, FCS(SA), CertPaedSurg(SA) \\ Rode H, FCS(SA), MMed(Chirg), FRCS(Edin)
}

Department of Paediatric Surgery, Red Cross War Memorial Children's Hospital, University of Cape Town

Correspondence: Prof H Rode, e-mail: Heinz.rode@uct.ac.za

\begin{abstract}
Although this article focuses predominantly on the management of paediatric burns, many of the principles and management protocols are universal and can be used for adults. Burns are defined as the coagulate destruction of tissue by thermal, chemical or electrical injury. This simplistic definition does, however, fail to incorporate the significant short- and long-term sequelae of these injuries, and the devastating social, functional and cosmetic consequences resulting from burn wounds.
\end{abstract}

(P) This article has been peer reviewed. Full text available at www.safpj.co.za

SA Fam Pract 2008;50(3):24-31

\section{Introduction}

Survival following a severe burn injury has improved substantially over the last 40 years. Although the overall morbidity is high, most patients with a burn wound of less than $50 \%$ total body surface area (TBSA) should survive. In experienced medical centres, even patients with burn wounds exceeding $70 \%$ have a good chance of survival. These results are based on refinements in the early phase of fluid resuscitation, and inhalational burn management. In the latter phases, results are improved by nutrition and bacterial control. Injuries are also surgically improved by a combination of early burn excision and early coverage by auto- or allografting (cadaver skin), biological and synthetic skin substitutes and lastly, by early and ongoing rehabilitation. Legislative requirements to reduce the hazards of thermal injury have also made an impact on the incidence and severity of burns, for example, lowering the geyser temperature to $55^{\circ} \mathrm{C}$.

\section{Prevalence}

Burn injuries affect $3,2 \%$ of South Africans annually and $50 \%$ of the patients are less than 20 years old. Burns are one of the commonest causes of traumatic death in children up to the age of four years and the third most common up to 18 years of age. These burns typically occur over weekends, at night and $75 \%$ occur at home. The most dangerous places are the kitchen and bathroom. Informal housing results in several factors placing children at risk. Close housing without fire breaks results in the rapid spread of fire. Open energy sources, like paraffin stoves or open fires, place the environment at considerable risk. Informal electrification with open wires and poor electrical safety places inquisitive children at risk. Storage of dangerous chemicals in beverage containers accessible to young children, e.g. cold drink bottles, makes ingestion likely. The majority of burns are minor and can be effectively treated by general medical and nursing practitioners. Approximately $11 \%$ would require hospitalisation in a burn unit.

\section{Aetiology}

The aetiology of burns varies according to age, activity and socioeconomic circumstances. Children are more likely to be burned under conditions of social disruption, whether this takes the form of poor socioeconomic infrastructure, poor adult supervision or blatant child abuse. It must be borne in mind that children are inquisitive by nature and their environmental exploration places them at risk for all types of burn injuries. Adult burns are due to fire injuries, motor vehicle accidents, industrial accidents, chemical mishaps and substance abuse.

The most frequent causes of burn injuries in children are summarised in table I.

Table I: Causes of burn injuriesas follows:

\begin{tabular}{|c|c|}
\hline Scalds & Boiling water, cooking oil, hot baths, hot beverages and food \\
\hline Flames & $\begin{array}{l}\text { House fires, open fires, paraffin stove explosions and } \\
\text { experimentation with fire (typically boys) }\end{array}$ \\
\hline Contact & Cooking appliances and heaters \\
\hline Electrical & $\begin{array}{l}\text { Low voltage (especially in informal developments) } \\
\text { with open wires } \\
\text { High voltage - overhead wires and substations }\end{array}$ \\
\hline Chemical & $\begin{array}{l}\text { Unsafe storage practices of household acids and alkalis, e.g. } \\
\text { stove cleaner, drain cleaner }\end{array}$ \\
\hline Sun & Over-exposure without appropriate sunscreen protection \\
\hline
\end{tabular}

Pathophysiology (Figure 1)

Local injury:

A burn results in three zones of injury. The central zone is a zone of necrosis that represents the area of maximum energy transfer and immediate cell death. This is surrounded by a zone of stasis that represents an area of impaired circulation and is potentially salvageable. The outer zone is a zone of hyperaemia where the least amount of energy transfer has occurred.

Systemic effects:

\section{Haemodynamic changes:}

Thermal injury alters endothelial integrity and function. The subsequent systemic inflammatory response syndrome (SIRS) is mediated through the widespread release of inflammatory mediators, primarily from the 
Figure 1: Jackson's burn model

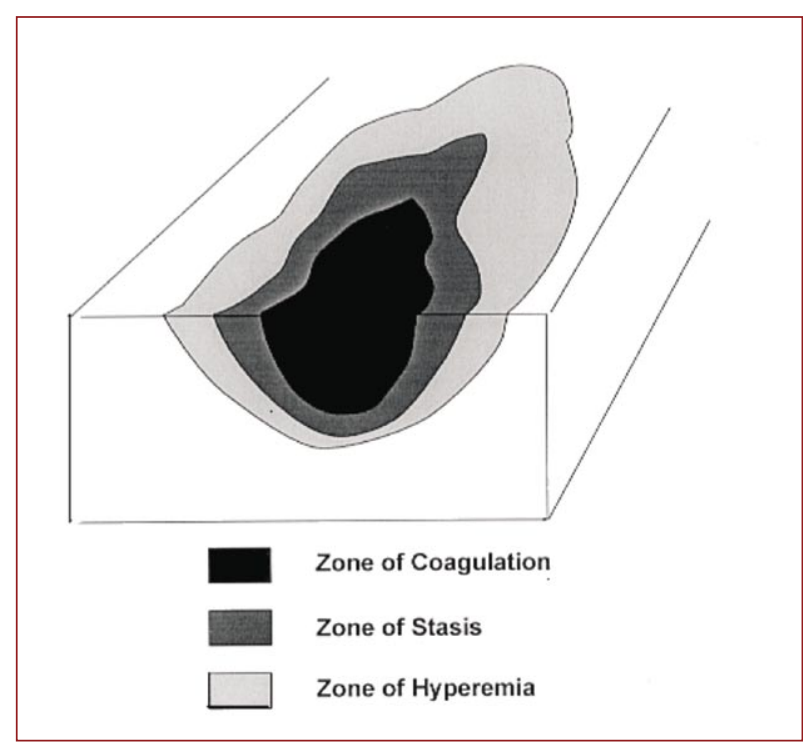

zone of hyperaemia. This results in obligatory isotonic fluid loss from the intravascular spaces into the burnt area. The capillary changes occur very rapidly and are maximal within the first 3-12 hours following small burns and up to 24-48 hours after larger burn injuries. This fluid loss can be as much as $4,4 \mathrm{ml} / \mathrm{kg} / \mathrm{hr}$. The fluid loss is not confined to the burnt area, but involves the whole body when the TBSA $>30 \%$. This obligatory sequestration of fluid will lead to the formation of oedema with eventual loss of effective circulating fluid volume. If this loss is extensive, hypovolaemia and shock will develop. It remains a common cause of death.

\section{Susceptibility to infection:}

The burnt patient is prone to microorganism invasion because of impaired local defense mechanisms (loss of outer skin barrier, presence of dead tissue and impaired local blood flow) and impaired systemic immune defenses due to a decrease in phagocyte and lymphocyte function, compounded by impaired humoral and cellmediated immunity. These factors together with an exposure to a high-risk environment for nosocomial infection and recurrent invasive procedures result in sepsis being the leading cause for delayed mortality among burn victims. Gram-positive organism infections predominate during the first week. Gram-negative and other organisms usually appear after five to seven days. Seventy per cent of the wounds harbour organisms from an exogenous source and $30 \%$ are from an endogenous source from the gastrointestinal, upper respiratory and from the genitourinary tract. The common organisms encountered are as follows: Staphylococcus aureus, Pseudomonas aeruginosa, Beta-haemolytic Streptococcus, Acinetobacter baumannii, Mixed Gram-negative flora, Enterococcus and Candida albicans. Unfortunately many of them have become resistant to topical antiseptics.

\section{Hypermetabolic response:}

Although the thermal insult occurs immediately, the systemic response to inflammatory mediators reaches a peak five to six days after the injury. Increased metabolic rates are often encountered in burns and may be as high as 50 to $100 \%$ above normal in major burns. This response is characterised by gluconeogenesis, severe protein catabolism, lipolysis and fat redistribution. Essential protein sources can be exhausted within three to five weeks, making nutritional supplementation essential either enterally or parenterally. Meticulous pain and infection control and an ambient temperature of 28 to $32{ }^{\circ} \mathrm{C}$ are all required to minimise metabolic expenditure.

\section{Renal failure:}

Several aspects may alter renal function, namely a low perfusion state due to under or delayed resuscitation, or alternatively, electrical or extensive burns may cause haemolysis, rhabdomyolysis with haemoglobinuria, myoglobinuria and renal failure.

\section{The gastrointestinal tract:}

The bowel is very susceptible to injury during the periods of hypovolaemia and lack of enteral feeding. More than $60 \%$ of patients may have evidence of bowel ischaemic injury at autopsy. Release of stress hormones induces mesenteric vasoconstriction and leads to decreased gut immune function and gut mucosal integrity, predisposing the patient to bacterial and endotoxin translocation of endogenous flora into the systemic circulation. This process can be ameliorated by early aggressive enteral feeding and adequate circulatory resuscitation.

\section{The musculoskeletal system:}

This system is often overlooked in a severely burnt patient. Two aspects are initially of great importance, namely the compartment syndrome and concomitant skeletal injuries that may be overwhelmed by the magnitude of the burn. The compartment syndrome is either due to a constricting full thickness eschar or alternatively, due to a swollen limb secondary to electrical injury and extensive underlying muscle damage. Signs may be subtle and pre-emptive decompression escharotomies may be required.

\section{Clinical}

All other things being equal, the following two aspects will determine further management and outcomes. An assessment of the burn wound area will determine fluid and metabolic needs and an estimation of the wound depth will determine local and surgical management.

\section{Estimation of total burn surface area (TBSA)}

The assessment of the burnt area is critical to the management of the patient and needs to be accurately determined. It is inherent to the successful fluid resuscitation and transfer protocols involved. Traditionally the 'Rule of Nine' is used for all patients older than 10 years (Figure 2). However, due to different body ratios this needs to be changed for a child. The child figure represents an infant up to one year. For every year thereafter the head decreases in relative size by approximately $1 \%$ and each leg gains $1 / 2 \%$. Thus adult proportions are gained at about 10 years of age. An alternative method to use is the patient's open hand that represents $1 \%$ of the body surface area. Pitfalls to the assessment of the surface area are electrical burns which have a greater area burned than what is evident on the surface.

\section{Estimation of burn wound depth}

The evolution in a burn wound is a dynamic process and it may take up to three to four days for the eventual depth to become evident. For clinical purposes there are only two types of burns (Figure 3).

\section{Those that heal spontaneously within two to three weeks:}

These wounds are epidermal burns or superficial partial thickness burns. Characteristically they are painful, weeping, and pink with blisters. They rarely give rise to functional or cosmetic deformities.

\section{Those that will not heal spontaneously:}

These are deep partial thickness or full thickness burns. They have a blotchy red discolouration, are less painful and may have either a weeping or dry surface. They may have small blisters. These wounds would require excision and grafting. They are prone to disfigurement and/or deformities. 
Figure 2: Body surface area distribution

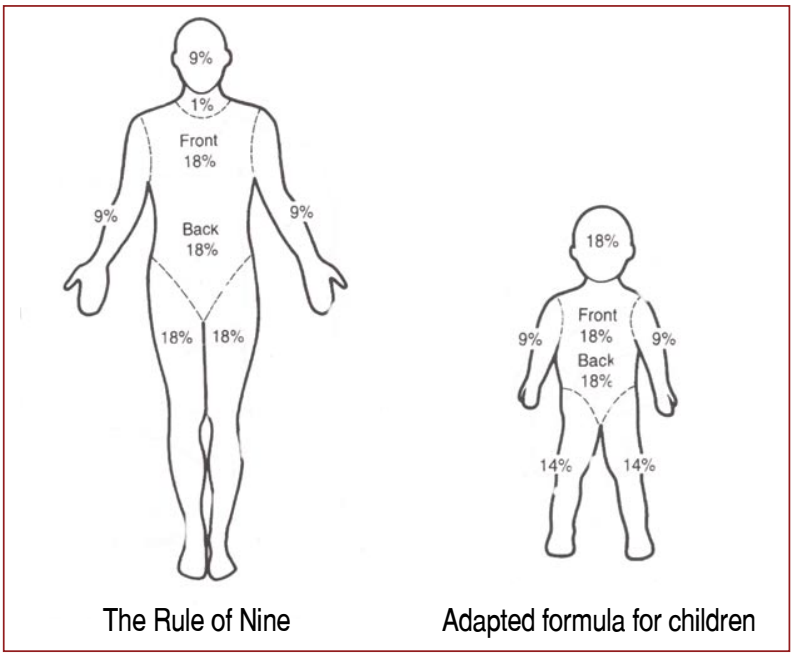

Acute Burn Management

Definitive management must occur during the first 24 hours. Immediately after first aid has been applied, the principles of primary and secondary surveys should be implemented.

\section{Primary survey}

A. Airway maintenance with cervical spinal control

B. Breathing and ventilation

C. Circulation with haemorrhage control

D. Disability and neurological status

E. Exposure and environmental control

F. Fluid resuscitation proportional to burn size

\section{A. Airway maintenance with cervical spinal control}

The detection of respiratory insufficiency is paramount during the initial examination as it is a major cause of early mortality. The cervical spine must be protected. Clear the airway by removing foreign material and ensure an open airway. All major burns must receive high flow oxygen for 24 hours.

The history of flame burns sustained in an enclosed space and loss of consciousness at the scene are good predictors of potential respiratory injury. Clinically the presence of burns on the face, either by flames or hot water, carbonaceous sputum or nasal discharge, a hoarse voice, confusion, stridor and finally, any of the routine signs associated with respiratory distress mandate very careful assessment, and early intervention. Signs of carbon

Figure 3: Burn depth and skin structure

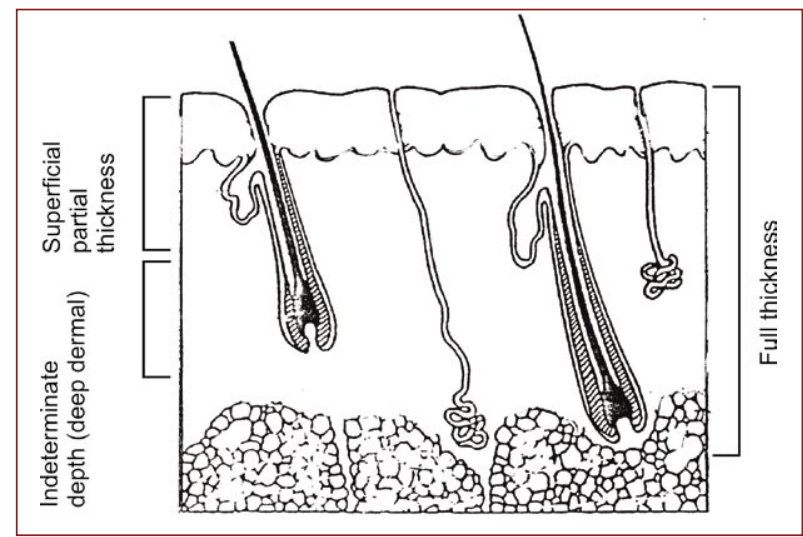

monoxide intoxication range from nausea and fatigue to confusion, seizures and coma. These patients characteristically look 'cherry red', a sign that is often absent, the pulse oximetery is misleading as carboxyhaemoglobin is mistaken for oxygenated haemoglobin - giving normal oxygen saturation readings. Hundred per cent oxygen should be administered via a non-rebreathing face mask.

\section{B. Breathing and ventilation}

Expose the chest and ensure that chest expansion is adequate and equal. Exclude associated injuries. Encasing eschars may restrict chest excursions and may necessitate early escharotomies. A respiratory rate of more than 20 per minute is a danger sign.

\section{Circulation with haemorrhage control}

A thorough assessment of the haemodynamic status should be made. Bleeding sites should be stopped with direct pressure. The pulse, capillary refill and blood pressure all need to be assessed (the blood pressure being the last of the indices to fall) on an ongoing basis. Large bore lines must be inserted preferably through unburned tissue, but can be through burn wounds if necessary. Intraosseous needles can also be used in younger patients.

\section{Disability and neurological status}

The level of consciousness should be determined. The patient could either be alert, responsive to vocal stimuli, responsive to painful stimuli or unconscious (AVPU).

\section{E. Exposure and environmental control}

The aim is to determine the body surface area burnt and the depth of the wound. All the clothes and jewellery should be removed. Children are at risk from developing hypothermia due to the large surface to mass ratio. Concomitant injuries must also be identified and managed.

\section{F. Fluid resuscitation proportional to burn size}

The patient should be assessed for shock and if present, a crystalloid fluid volume of $20 \mathrm{ml} / \mathrm{kg}$ of Ringer's lactate solution should be given immediately. This should be followed up until the shock has been corrected. Fluid therapy thereafter consists of two components, namely ongoing fluid loss replacement and maintenance fluid that in children should contain $5 \%$ glucose.

Ongoing fluid requirements need be calculated as follows if > 10\% TBSA:

Resuscitation (Ringer's lactate solution)

- Day $1: 3 \mathrm{ml} \times \mathrm{kg} \times \%$ burn. First half to be given in the eight hours from the time of injury and the second half in the subsequent 16 hours.

- Day 2: $2 \mathrm{ml} \mathrm{x} \mathrm{kg} \mathrm{x} \mathrm{\%} \mathrm{burn} \mathrm{over} 24$ hours

\section{PLUS}

Maintenance (paediatric maintenance solution - not half-strength Darrows/Dextrose solution)

- $100 \mathrm{~m} / \mathrm{kg}$ up to $10 \mathrm{~kg}$ plus

- $50 \mathrm{~m} / \mathrm{kg}$ from $10-20 \mathrm{~kg}$ plus

- $20 \mathrm{~m} / \mathrm{kg}$ for each $\mathrm{kg}$ over $20 \mathrm{~kg}$

The above is only a guide and is used to maintain urine output at $1 \mathrm{ml} /$ $\mathrm{kg} / \mathrm{hr}$. If urine output is low and perfusion poor, then give a fluid bolus of $10 \mathrm{ml} / \mathrm{kg}$ or increase the fluid resuscitation to $150 \%$ of the previous hour and reassess. 


\section{First aid}

The burning process should be interrupted as soon as possible. Remove smouldering or hot clothing immediately and ensure an open airway. The burn wound should be cooled with running, cold tap water at $16{ }^{\circ} \mathrm{C}$ for $20-30$ minutes. Ice should not be used as it may deepen the burn. In large burn wounds caution must be taken not to induce hypothermia of the patient. Copious irrigation with water must be used for chemical burns until the pain has disappeared. Burnshield (Melaleuca Alternifolia Hydrogel) can be applied de novo or following cooling and the wound can then be dressed with a clean dressing or cling wrap followed by a light crepe bandage to hold the dressings in place. Cooling serves to halt the burning process and prevents the area of coagulation from spreading, thus limiting the size and depth of the burn. It can still be effective up to three hours after the injury.

\section{Pain relief}

Burns are exceptionally painful and apart from the humane value of providing analgesia, it serves to diminish the systemic inflammatory reaction, by modulating the sympathetic response and hence cytokine secretion (Table II). It also serves to diminish long-term psychosocial scarring. There are three components to be considered, namely acute, procedural and chronic pain. Pain management is an integral part of the programme and must be initiated from the beginning.

Table Il: Analgesics and anxiolytics

\begin{tabular}{|c|c|c|}
\hline $\begin{array}{l}\text { Acute } \\
\text { management }\end{array}$ & $\begin{array}{l}\text { Morphine } \\
\text { Tilidine } \mathrm{HCl}\left(\text { Valoron }^{\circledR}\right) \\
\text { Paracetamol }\end{array}$ & $\begin{array}{l}10-40 \mathrm{mcg} / \mathrm{kg} / \mathrm{hr} \text { infusion IVI } \\
0,5-1 \mathrm{mg} / \mathrm{kg} / \mathrm{dose} \\
20 \mathrm{mg} / \mathrm{kg} / \mathrm{dose}\end{array}$ \\
\hline Sedation & $\begin{array}{l}\text { Trimeprazine }(\text { Vallergan } \\
\text { Droperidol }\left(\text { Inapsin }^{\circledR}\right) \\
\text { Hydroxyzine }(\text { Aterax }\end{array}$ & $\begin{array}{l}2-3 \mathrm{mg} / \mathrm{kg} / \mathrm{dose}(\max 100 \mathrm{mg}) \\
0,1-0,2 \mathrm{mg} / \mathrm{kg} / \mathrm{dose}(\max 5 \mathrm{mg}) \\
0,5-1 \mathrm{mg} / \mathrm{kg} / \mathrm{day}\end{array}$ \\
\hline Anxiolysis & Midazolam Dormicum ${ }^{\circledR}$ ) & $0,5 \mathrm{mg} / \mathrm{kg} /$ dose $(\max 15 \mathrm{mg})$ \\
\hline $\begin{array}{l}\text { Changing of } \\
\text { dressing }\end{array}$ & $\begin{array}{l}\text { Ketamine } \\
\text { Codeine } \\
\text { Brufen } \\
\text { Paracetamol }\end{array}$ & $\begin{array}{l}\text { IVI 0,25-0,5 mg/kg/dose } \\
\text { IMI } 1-2 \mathrm{mg} / \mathrm{kg} / \mathrm{dose} \\
\text { Oral } 0,5 \mathrm{mg} / \mathrm{kg} / \text { dose } \\
0,5-1 \mathrm{mg} / \mathrm{kg} / \mathrm{dose} \\
5-6 \mathrm{mg} / \mathrm{kg} / \mathrm{dose} \\
15-20 \mathrm{mg} / \mathrm{kg} / \mathrm{dose}\end{array}$ \\
\hline
\end{tabular}

\section{Secondary survey}

A general history including details of the mechanism of the injury and pre-existing diseases and allergies should be recorded, as well as a repeated examination of the patient from head to toe. If transfer is contemplated, consent and the necessary documentation including medical records, calculated fluids and medications, urine output and vital signs must accompany the patient.

\section{Special Investigations}

Investigations are directed toward monitoring the efficacy of resuscitation, and the multiple changes in the haematological, biochemical and bacteriological spheres during the emergent period. Radiology will include the cervical spine and the pelvis. Laboratory investigations include carboxyhaemoglobin levels, acid base status and lactate, all of which will provide evidence of end organ perfusion. A urine examination for concentration, specific gravity and myoglobin will aid the resuscitation process.

\section{Transfer Procedures}

Burn patients are usually stable immediately after injury and transfer can be safely undertaken once initial therapy has been instituted, the patient stabilised and a decision made about which facility or hospital the patient should be transferred to.
All escharotomies identified in the secondary survey should ideally be performed prior to transfer. Early consultation for advice and transfer are essential in the management of the burns patient.

Transfer is indicated for the following:

- Patients with burns greater than $5 \%$ TBSA

- Burns of special interest - face, hands, feet, genitalia, perineum and major joints

- Electrical burns

- Chemical burns

- Inhalation injury

- Circumferential burns

- Pre-existing medical conditions

- Associated trauma

- Suspected child abuse

- Any patient that cannot be managed at the referring facility

Upon transfer to a burns centre it is important to do the following:

Clearly document the history and time of the burn, the fluids planned and received and a diagrammatic representation of the burned area. Send consent for repeated excision, grafting, change of dressing and splinting under anaesthetic. Ensure a safe transport method and qualified accompanying personnel to continue resuscitation en route.

\section{Dressings}

Small superficial or partial thickness wounds can be treated with a semi-permeable occlusive dressing, e.g. Omiderm ${ }^{\circledR}$ and left until healed, or a hydrocolloid dressing, e.g. Granuflex and changed every four days. Large or deep burns require antiseptic dressings and definitive surgical management at a burns centre. Options for antiseptic dressings include the following:

- Silver sulfadiazine (Flamazine ${ }^{\circledR}$ ): This antiseptic has an excellent spectrum of activity with low toxicity, and ease of application with minimal pain. It is effective for at least 24 hours. The use of silver sulfadiazine is frequently associated with the development of a 'pseudo-eschar' within two to four days, which can lead to errors in the evaluation of burn depth by the inexperienced observer.

- Povidone iodine (Betadine ${ }^{\circledR}$ ): Povidone has a wide antibacterial spectrum and is inactivated by wound exudate. It has a half-life of 12 hours and needs to be applied twice daily.

- Mupirocin (Bactroban ${ }^{\circledR}$ ): It has excellent broad spectrum antimicrobial activity, predominantly against Gram-positive organisms and Methicillin resistant Staphylococci (MRSA). It is insensitive to Pseudomonas and can be applied daily.

- Chlorhexidine (Hibitane ${ }^{\circledR}$ ): Chlorhexidine has a broad spectrum anti-microbial action, effective especially against Pseudomonas infection. It is often combined with Mupirocin to broaden the antibacterial spectrum. It should be changed daily.

- Acticoat: Nanocrystalline silver has made a significant difference to the treatment of burn wounds. It liberates silver $\left(\mathrm{AG}^{\circ}\right)$ at a concentration ten times the MIC for more than 150 organisms. It is applied topically and removed every third to fourth day.

\section{Definitive Management}

\section{Minor burns}

Initial therapy of minor burns should include administration of analgesics and cleaning of the wound. Dead tissue should be debrided and any tar removed with soft paraffin in a water base or mineral oil. Topical antibacterial agents and occlusive dressings or an adhesive polyurethane sheet should be used to dress the wound (Omiderm ${ }^{\circledR}$ ) 
and tetanus toxoid should be administered. The patient should be encouraged to move the affected area. Local topical therapy is applied every two to three days until the wound is healed or Omiderm ${ }^{\circledR}$ is left undisturbed for seven to ten days or until the wound has healed. Prophylactic antibiotics are usually not required. The wounds should be examined regularly (daily/once a week) as necessary. Wounds that fail to heal within three weeks will need to have a skin graft.

\section{Major burns}

Management of major burns is a particularly time and energy intensive process and the requirement of a coherent multidisciplinary team cannot be underestimated. Burns involving the superficial dermis heal within three weeks with little or no hypertrophic scarring. Primary skin grafting will not decrease morbidity or improve the functional outcome. On the other hand, full thickness burns should be excised and grafted. Many burns, however, cannot be easily categorised. The decision to excise and graft is therefore crucial. The principles of burn surgery include the following:

- Burns in the very young and aged are usually not superficial.

- Burn wounds from flames tend to be deeper and of greater severity than hot water (scald) burns.

- Contact burns of the soles and palms usually heal within three weeks.

- Electrical burns must be considered as deep and flash burns as more superficial.

- Superficial and partial thickness thermal injuries treated correctly will heal spontaneously within three weeks, can be clinically identified and are best treated conservatively.

- Hot water (scald) burns in children are best left for two weeks to assess the need for operative intervention, thereby reducing the area for excision by $>50 \%$.

- Surgery is an elective procedure in a stable patient.

- Early excision and grafting should be considered the treatment of choice for all full thickness burns. Inadequate excision leads to skin graft loss. Exceptions might be ears, soles of the feet, genitalia and the face.

- The amount to be excised depends on the status of the patient, the burn size, availability of auto- or allografts or skin substitutes and the volume of blood loss.

- Once the burn wound has been excised, immediate wound closure with autografts, allografts or biological alternatives is required.

- Burns that are not life-threatening in patients with severe concomitant diseases or injuries should only be excised when the patients are stable and the life-threatening processes controlled.

- Surgical excision can safely be done in the presence of inhalational injury.

Beta-haemolytic Streptococcus infection, and heavily contaminated untreated wounds are a contraindication to surgical excision and grafting should be delayed until the infection has been eradicated.

\section{Theatre Management}

Vascular access must be adequate and secure. Large bore cannula access is preferred; a central venous catheter may often be the only available option. Arterial lines are invaluable, and often provide the only continuous monitoring available during surgery on major burns.

Temperature control is mandatory, as hypothermia is a significant problem. Preventable measures include an ambient theatre temperature of $28-32{ }^{\circ} \mathrm{C}$, warmed anaesthetic gases and intravenous fluids, the use of overhead radiant heaters, and a plastic-covered warming blanket on the operating table. Exposed areas may be covered with sterile plastic drapes.
Monitoring is a challenge. ECG electrodes may be placed on the chest, but it may be necessary to use the hands, feet, forehead, back or any other available dry surface. Surgical staples in the skin with crocodile clips attached are very useful. A urinary catheter is useful but it frequently crosses the field of surgery, and may be temporarily plugged off during the procedure.

\section{Surgical guidelines}

The process of excision, grafting and progressive burn wound coverage must follow a predetermined progression. There will, however, need to be flexibility based on the healing process.

The theatre procedure follows a set programme:

- Administer anaesthesia.

- Clean the wound with bland soap and water or detergent.

- Remove any topically applied agents.

- To be able to evaluate the extent of the burn, shave the hair where necessary.

- Dead tissue should be debrided.

- Joint and burn mobilisation by allied therapists.

- The depth and extent of the burn wound will determine the surgical approach.

- Excise and graft as per treatment plan.

- Dress the wound with topical antibacterial agents, occlusive dressings, a non-adhesive polyurethane sheet or temporary skin substitutes where indicated.

- Routine antibiotics are not required.

The amount to be excised at each procedure depends on the stability of the patient, the burn size, availability of auto- and allografts, the volume of blood loss incurred during the procedure and the adequacy of anaesthesia.

\section{Surgical management}

Five surgical procedures are utilised, i.e. decompression escharotomies, tangential excision, fascial excision, delayed escharectomy and skin grafting.

\section{Decompression escharotomies}

Decompression escharotomies are emergency procedures done at the time of first assessment of the patient. Oedema accumulates under the rigid burned skin that acts as a tourniquet, thereby obstructing normal circulation. This is part of resuscitation and should be performed as soon as possible to avoid irreversible ischaemic and hypoxic damage of tissue. Releasing decompression must be done where a circumferential deep burn is impeding circulation to more distal parts, especially round the arms, legs, or chest where respiration may be impaired.

The longitudinal decompression incision must traverse the dead tissue as far into the subcutaneous layer as necessary to encounter viable tissue, and must extend from non-viable through to viable tissue. Bleeding may be a problem, especially if the incisions are too deep or performed across major vessels. Vital structures (e.g. nerves, blood vessels) may be damaged if the decompression is incorrectly sited. Transverse incisions in the limbs should not be made. Correct placement of these escharotomies is essential to avoid problems during the reconstructive and rehabilitation phases of treatment. Anaesthesia may not always be necessary for the decompression procedures, but monitor the response to surgery, and provide anaesthesia and analgesia if this becomes necessary.

Tangential or sequential excision

This entails the sequential excision of thin layers of burnt eschar until 
a viable bed is encountered. This technique requires considerable experience and theatre facilities. The appropriate level of excision or end-point is characterised by a shiny white surface with a brisk arteriolar or punctuate bleeding, or viable yellow non-haemorrhagic subcutaneous fat globules with bleeding vessels in all areas. Dark pink-brown haemorrhagic fat is non-viable and must be removed, as residual necrotic areas will jeopardise graft take. Tangential excision is best done within the first three to five days, before hypervascularity and wound infections have become established. Blood loss may be substantial, and effective control of haemorrhage must be established. A maximum of $\pm 20 \%$ TBSA should be excised at any one time. Once excised to the appropriate level and haemostasis secured, an immediate split-thickness skin graft must be performed. Sheet grafts are placed on important cosmetic and functional areas.

\section{Fascial excision}

This procedure is generally reserved for very large, life-threatening, or deep full thickness burns. The excision is preferably limited to approximately 10-20\% TBSA at any one time. Fascial excision assures a viable bed for skin grafting with moderate blood loss, especially if done under tourniquet control. Excellent graft take may be expected if it is done within the first few days after injury. If a primary skin graft cannot be performed, consider vacuum assisted closure (VAC) or temporary skin substitutes. The major disadvantage of this method is that it causes damage to lymphatics, cutaneous nerves, loss of subcutaneous fat, long-term cosmetic deformity, and distal oedema.

\section{Delayed escharectomy}

This is done after one week or later, or after spontaneous eschar separation has allowed for the formation of a bed of granulation tissue. Daily debridement by means of hydrotherapy (showering or bathing), or coarse mesh gauze dressings will hasten eschar separation. The burn wound is ready for split skin grafting when there is a shiny, slightly granular, pinkish-red uniform bed of granulation tissue with no debris or evidence of infection. This method is most often used for old neglected burn wounds.

\section{Haemostasis}

Bleeding can be brisk or profuse and should be minimised. Methods employed are local pressure for 10 minutes, diathermy or bipolar coagulation, suture ligation and the topical application of sponges soaked in $1: 10000 / 1: 30000$ epinephrine (adrenaline) solution to the excised bed for 10 minutes.

\section{Skin grafting}

Procured skin is grafted onto the recipient area at the time of eschar excision, directly from the mesh board. Grafts are placed with the shiny or cut surface facing the prepared bed, either longitudinally or transversely over joints. The edges should be approximated or slightly overlapping and secured with surgical clips, sutures, or fine mesh gauze. Alternatively, small to moderate-sized skin grafts can be secured directly with adhesive dressings or glue and covered with a bandage. In general terms the recipient area is covered with an occlusive dressing to prevent infection, avulsion and desiccation of the graft, and to allow for graft vascularisation.

Donor sites may be restricted in a burn more than 40\% TBSA. As all donor sites scar to some degree, it is preferable to take skin from an area that can be hidden under most circumstances. The donor site for skin grafts needs to be treated as a partial thickness burn. Initially bleeding is stopped with epinephrine swab compression. Then, dressings are placed with either antiseptics or occlusive dressings.
Poorly cared for or infected donor sites can become deep and hence extend the total wound area.

\section{Wound closure}

If the burned area is too large, with limited available donor sites, temporary closure by another form of covering is indicated to provide a physiological and mechanical barrier while healing takes place. Allograft or skin donated by another person/cadaver is the most commonly used skin alternative. When applied to a clean excised wound, the tissue vascularises and provides cover until it is recognised as foreign by the host, resulting in graft loss approximately three weeks after application. Other material that can be used include Biobrane ${ }^{\circledR}$.

\section{Nutrition}

Patients with $>20 \%$ TBSA require aggressive nutritional support and the calorie and protein requirements should be met from the first day to prevent impaired wound healing, cellular dysfunction and decreased resistance to infection. Early enteral feeding within the first 12-24 hours post-burn decreases the release of stress hormones, improves nitrogen balance, maintains gut mucosal integrity, lowers the incidence of diarrhoea and decreases the hospital stay.

Due to high feeding requirements patients often require tube feeds either via a nasogastric route, or if not tolerated, by a naso-jejunal tube. The oral route is possible but difficult to sustain, especially when combined with interrecurrent episodes of sepsis and theatre visits. Total parenteral nutrition has been associated with infections, metabolic and immunological complications.

\section{Physical And Occupational Therapy}

Involvement of therapists in the theatre team is essential. It provides an opportunity to maintain joint mobility, and to apply and construct splints that will be crucial to the long-term functional outcome of a patient. Early splinting in combination with pressure garments and goal-directed physiotherapy will reduce complication rates and joint contractures that may limit rehabilitation.

\section{Rehabilitation}

Following a burn, a sequence of events is precipitated in the family. The family reacts with shock, anxiety, confusion and guilt. Eventually they take control of the situation that fluctuates between confidence, guilt, hope, doubt and often withdrawal and eventually they come to terms with the consequences. They are emotionally drained and concerned about the future of the burnt patient. They are often doubtful and sceptical about the process and outcome of rehabilitation. This programme can continue for more than 10 years and should only stop once all physical and psychological rehabilitation aspects have been completed. The involvement of psychologists, physical and occupational therapists and reconstructive teams are invaluable. The success of any burn programme should probably best be measured by the successful reintegration of its patients into society rather than its ability to preserve life.

\section{Conclusion}

The successful management of burns requires a dedicated multidisciplinary team of individuals. Following on the use of the principles of early resuscitation and maximum tissue preservation completed by early wound coverage, while supporting the patient systemically and psychologically can ultimately lead to a successful outcome in these potentially devastating injuries.

References: Available on request from the authors. 